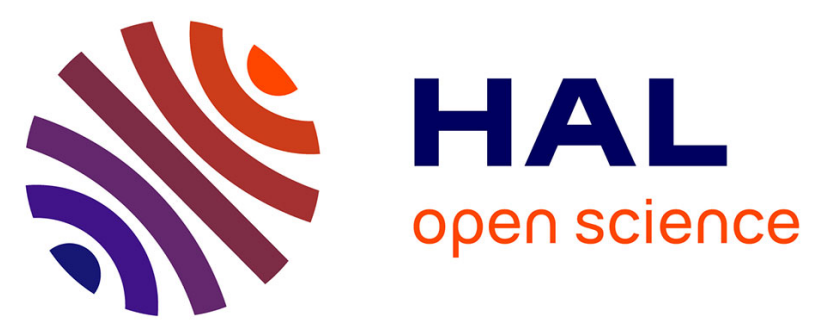

\title{
Analytical, numerical and experimental study of the bifurcation and collapse behavior of a 3D reinforced sandwich structure under through-thickness compression \\ Cyril Laine, Philippe Le Grognec, Sébastien Comas-Cardona, Christophe Binetruy
}

\section{To cite this version:}

Cyril Laine, Philippe Le Grognec, Sébastien Comas-Cardona, Christophe Binetruy. Analytical, numerical and experimental study of the bifurcation and collapse behavior of a 3D reinforced sandwich structure under through-thickness compression. International Journal of Mechanical Sciences, 2013, 67, pp.42 - 52. 10.1016/j.ijmecsci.2012.12.005 . hal-01666055

\section{HAL Id: hal-01666055 https://hal.science/hal-01666055}

Submitted on 18 Dec 2017

HAL is a multi-disciplinary open access archive for the deposit and dissemination of scientific research documents, whether they are published or not. The documents may come from teaching and research institutions in France or abroad, or from public or private research centers.
L'archive ouverte pluridisciplinaire HAL, est destinée au dépôt et à la diffusion de documents scientifiques de niveau recherche, publiés ou non, émanant des établissements d'enseignement et de recherche français ou étrangers, des laboratoires publics ou privés. 


\title{
Analytical, numerical and experimental study of the bifurcation and collapse behavior of a 3D reinforced sandwich structure under through- thickness compression
}

\author{
Cyril Lainéa ${ }^{\mathrm{a}}$ Philippe Le Grognec ${ }^{\mathrm{a}}$, Sébastien Comas Cardona ${ }^{\mathrm{a}, \mathrm{b}}$, Christophe Binetruy ${ }^{\mathrm{a}, \mathrm{b}}$ \\ ${ }^{a}$ Ecole des Mines de Douai, Polymers and Composites Technology E Mechanical Engineering Department, 941 rue Charles Bourseul - BP 10838,59508 Douai Cedex, France \\ ${ }^{\mathrm{b}}$ LUNAM Université, Ecole Centrale de Nantes, Research Institute in Civil Engineering and Mechanics (GeM), 1 rue de la Noë - BP 92101, 44321 Nantes Cedex 3, France
}

Three-dimensional reinforced sandwich structures are generally designed in order to provide optimal out-of-plane mechanical properties, beside traditional sandwiches which generally display satisfactory specific flexural properties but fatally insubstantial stiffnesses in the through-thickness direction. This paper deals with sandwich structures manufactured with polymeric foam core reinforced thanks to the Napco ${ }^{\mathbb{R}}$ technology (which is based on transverse needling) and specially investigates the compression behavior through the thickness direction. The compressive longitudinal moduli of such sandwiches are shown to be significantly improved in the presence of fibrous orthogonal reinforcements but the experimental estimations rather differ from analytical predictions that can be easily obtained using classical mixture laws. These discrepancies are mainly due to the buckling phenomenon, inherent in the geometry of the reinforcements, and the associated imperfection sensitivity. This work is thus devoted to the prediction of the critical loading of such a sandwich structure under through-thickness compression. A closed-form analytical expression is first derived from a general 3D elastic bifurcation analysis, then compared to finite element numerical solutions (obtained by both linearized buckling and incremental post-buckling analyses) for validation purposes, and finally confronted to experi-mental results. The buckling phenomenon is actually proved to be mainly responsible for the final collapse of the through-thickness reinforced sandwich.

Keywords: Buckling, Reinforced sandwich, Analytical solution, Finite element simulation, Periodic structure, Compression test

\section{Introduction}

Sandwich materials are commonly used in many applications of aerospace, marine or transportation industries, among others, due to the attractive combination of a lightweight and strong mechanical properties. The flexural stiffness of classical sandwiches is indeed particularly significant, thanks to the high strength of the skins and their distance from the middle-surface of the structure. However, the principal weakness of such classical sandwiches turns out to be the out-of-plane behavior, directly related to the low mechanical properties of the homogeneous soft core material. In order to improve the load carrying capacity in the thickness direction (in transverse shear and compressive behaviors) without being detrimental to lightness, the low density core layer is usually strengthened by appropriate reinforcements. As far as the compressive modulus is concerned, the simplest way to proceed comes down to add orthogonal reinforcements embedded in the upper and lower skins. Among the existing methods, such as tufting, Z-pinning and stitching [1], the patented Napco ${ }^{\mathbb{R}}$ technology, which is based on transverse needling, allows one to produce tailored sandwich structures in a continuous way, while preserving a high production efficiency and a relatively low cost.

The main objective of this study is to analyze the mechanical behavior of such Napco ${ }^{\circledR}$ sandwich structures under throughthickness compression. For this purpose, experimental compression tests are first performed in order to assess the significant increase of the equivalent compressive modulus due to the presence of reinforcements. In every instance, the experimental effective compressive moduli are unexpectedly small and scattered in comparison with the analytical predictions based on mixture laws, as already mentioned in Guilleminot et al. [2] These discrepancies are not due to the inadequacy of the mixture laws which perfectly apply in such a geometric configuration, but may rather be attributed to the imperfection-sensitive local buckling of the cylindrical reinforcements which may lead to collapse. Therefore, the present work aims at estimating the 
corresponding critical loading in order to predict the occurrence of buckling and further optimize the size and volume fraction of reinforcements in relation to the compression behavior.

Due to the geometry of reinforcements, a compressive loading in the thickness direction of such a 3D reinforced sandwich will fatally lead to an instability phenomenon. The buckling response of the composite material can be seen as the buckling of reinforcements (being considered as slender beams) inside the homogeneous core material. When the core material is neglected, the buckling of reinforcements is governed by Euler's theory, as observed by Lascoup et al. [3] in the context of stitched sandwich structures under transverse compression and out-of-plane shear. In practice, despite a comparatively low modulus, the core material strongly influences the critical buckling load of the reinforcements (displaying a stabilizing effect), and the classical Euler critical values, obtained without any core material, are generally no more valid at all. Several analytical solutions have already been proposed in the literature to better estimate the critical loading for such a micro-buckling problem, which is considered as a major collapse mode in fiber-reinforced composite materials. The pioneering works date back to the sixties and were conducted by Rosen. A very simple expression is suggested for the buckling value, but the strong hypothesis of a uniform shear state in the matrix material restricts its validity domain. From this point, other simplified solutions have been deduced among which the one from Drapier et al. [4]. These authors considered a single unidirectional ply of a laminated composite and calculated the buckling load using an homogenization technique and assuming a simplified strain state in both reinforcements and matrix. Few authors, among which Liu et al. [5], interested in the buckling of a sandwich structure reinforced in the thickness direction, similar to the Napco ${ }^{\circledR}$ sandwich. In their analytical approach, the through-thickness reinforcements are modeled by simply supported beams and the continuous core material is replaced by the superimposition of horizontal and vertical elastic spring distributions. This representation is thus a generalization of the model initially introduced by Timoshenko in the context of the buckling of beams relying on elastic foundations. None of the simplified solutions identified in the literature, including the two previously mentioned, provides satisfactory results from a general point of view. Some of them are in good agreement with reference numerical (or experimental) results provided that specific conditions are fulfilled (for instance, the volume fraction of reinforcements is particularly low or high). As an alternative, some authors have searched out "exact" solutions, considering the core material like a continuous surrounding medium, without any further simplification regarding the deformation field. The most numerous results available in the literature concern composite materials made of long fibers and are obtained in a 2D modeling space. Parnes and Chiskis [6] provided such an exact solution in the context of nano-filled polymer composites and compared their own results with the values obtained by Rosen's formula. Sooner, Zhang and Latour [7] showed that the prevailing buckling mode was the so-called shear mode, as long as the volume fraction of reinforcements is sufficiently high, such that reinforcements interact with each other. A closed-form solution may be obtained in a 2D framework, whereas only a semi-analytical solution is conceivable when considering the real 3D geometry. In Zhang and Latour [8], the same authors derived such an approximate solution partly based on numerical computations of a 3D unit cell made of a cylindrical reinforcement surrounded by the matrix. They compared the results from both $2 \mathrm{D}$ and $3 \mathrm{D}$ models and tried to define the best 2D representation of the reinforced material, in terms of geometric dimensions and volume fractions, which would be capable of displaying the same critical values as in $3 \mathrm{D}$.
In this study, the objective is to solve analytically the buckling behavior of the Napco ${ }^{\circledR}$ sandwich under through-thickness compression. A 2D model is defined with the appropriate boundary conditions in order to derive a closed-form analytical solution. The foam core is represented as a continuous medium whereas a beam model is considered for the reinforcements. The skins are not directly modeled but their presence is taken into account through the boundary conditions applied to the reinforced foam core. Some conventions are specially suggested in order to relate properly the 2D designed model to the real 3D configuration. Numerical finite element computations are then performed on a 3D unit cell of the sandwich material in order to validate the analytical modeling. All these predictive solutions are shown to be in good agreement with the experimental force-displacement curves plotted from a series of compression tests. It allows one to state that the micro-buckling of the reinforcements is the main reason for the collapse of the sandwich structure when it is compressed through the thickness direction.

\section{Experimental data}

\subsection{Napco $^{\circledR}$ technology}

The Napco ${ }^{\circledR}$ technology is a manufacturing process of 3D sandwich composites based on transverse needle punching. It consists in strengthening the foam core of a sandwich structure by adding orthogonal (or inclined) through-thickness reinforcements in order to particularly enhance some of the mechanical properties. It differs from other technologies such as stitching due to the fact that the fibrous reinforcements here come from the skin material, so that the facing fabrics (mats) and the foam core make up a monolithic whole (see Fig. 1). In practical terms, a set of needles regularly penetrates the sandwich structure on both sides, according to the desired pattern and density, the needles catching and carrying yarns from the facings through the core material, as shown in Fig. 2. Once the 3D sandwich preform is produced, it is impregnated by a liquid resin. Among the different liquid composite molding techniques, the VARIM process (Vacuum Assisted Resin Infusion Molding) has been retained for its efficiency.

The creation of the fibrous reinforcements and the composite manufacturing, associated with an experimental campaign of measurement of geometric and material parameters, lead one to a realistic and optimal representation of the sandwich architecture and thus to a proper prediction of the effective mechanical properties when using appropriate analytical or numerical tools.

\subsection{Geometric and material data}

The 3D sandwich samples which will be subsequently tested are made up of a linearly elastic isotropic closed cell polyurethane foam (whose density is $40 \mathrm{~kg} \cdot \mathrm{m}^{-3}$ ). Both facings are made of one ply of chopped strand glass mat and one carbon [0,90] cross-ply laminate. During the infusion process, use is made of an Epolam 5015 epoxy resin with $20 \%$ of 5015 hardener. The material data are summarized in Table 1.

The skins are supposed to be isotropic, with equivalent Young's modulus $E_{s}$ and Poisson's ratio $v_{s}$. The cylindrical fibrous reinforcements are perpendicular to the skins and viewed as unidirectional composite columns (UDs) composed of aligned isotropic fibers surrounded by resin (see Fig. 3). Since the micro-buckling of fibers inside the reinforcements is out of the scope of this paper and thus ignored in our future model, the heterogeneous fibrous reinforcements can simply be represented by equivalent homogeneous cylinders. A preliminary 
homogenization step, based on advanced mixture laws [9], is then first performed, involving the volume fraction of the fibers within the reinforcements $V_{f}$ (obtained through burn off tests) and the material properties of both constituents (glass fibers and resin). It gives the following equivalent properties for the transversely isotropic through-thickness reinforcing composites (due to the unidirectional arrangement of the fibers):

$$
\begin{aligned}
& E_{L}=E_{f} V_{f}+E_{r}\left(1-V_{f}\right) \\
& v_{L T}=v_{f} V_{f}+v_{r}\left(1-V_{f}\right) \\
& G_{L T}=G_{r} \frac{G_{f}\left(1+V_{f}\right)+G_{r}\left(1-V_{f}\right)}{G_{f}\left(1-V_{f}\right)+G_{r}\left(1+V_{f}\right)} \\
& G_{T}=G_{r}\left(\begin{array}{c}
1+\frac{V_{f}}{\frac{G_{r}}{G_{f}-G_{r}}+\frac{k_{r}+\frac{7 G_{r}}{3}}{2 k_{r}+\frac{8 G_{r}}{3}}\left(1-V_{f}\right)}
\end{array}\right)
\end{aligned}
$$

a

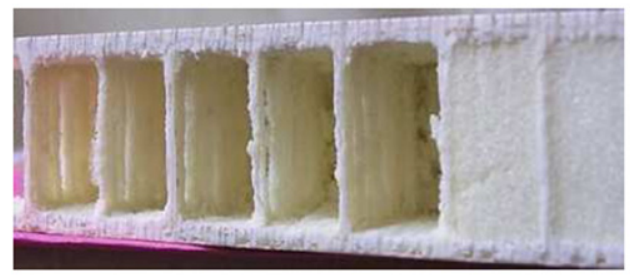

C

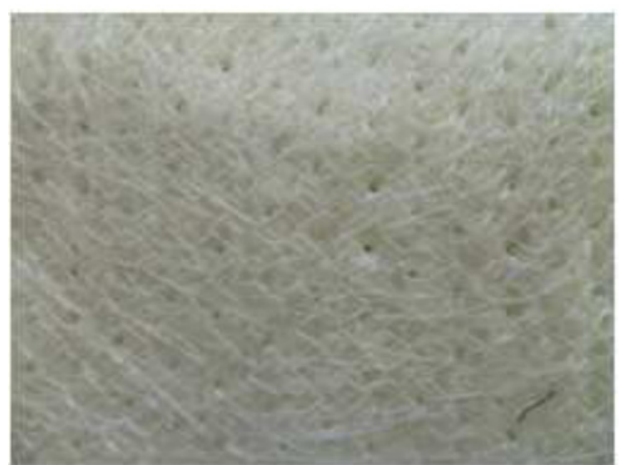

$$
\begin{aligned}
& K_{L}=K_{r}+\frac{1}{\frac{1}{k_{f}-k_{r}+\frac{G_{f}-G_{r}}{3}}+\frac{1-V_{f}}{k_{r}+\frac{4 G_{r}}{3}}} \\
& E_{T}=\frac{2}{\frac{1}{2 K_{L}}+\frac{1}{2 G_{T}}+\frac{2 v_{L T}^{2}}{E_{L}}} \\
& v_{T}=\frac{E_{T}}{2 G_{T}}-1
\end{aligned}
$$

where the quantities $E_{i}, v_{i}, G_{i}, k_{i}$ and $K_{i}$ represent Young's moduli, Poisson's ratios, shear moduli, bulk moduli and transverse bulk moduli (without longitudinal strain), respectively, and the subscripts

Table 1

Material properties.

\begin{tabular}{lllll}
\hline Material & $\begin{array}{l}\text { Polyurethane } \\
\text { foam }\end{array}$ & $\begin{array}{l}\text { Epoxy } \\
\text { resin }\end{array}$ & $\begin{array}{l}\text { Glass } \\
\text { fiber }\end{array}$ & $\begin{array}{l}\text { Carbon } \\
\text { fiber }\end{array}$ \\
\hline $\begin{array}{l}\text { Young's modulus (MPa) } \\
\text { Poisson's ratio }\end{array}$ & 6.7 & 3281 & 72400 & 290000 \\
& 0.001 & 0.35 & 0.22 & 0.3 \\
\hline
\end{tabular}

b

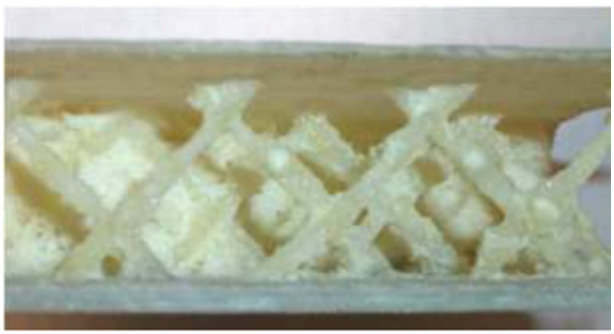

d

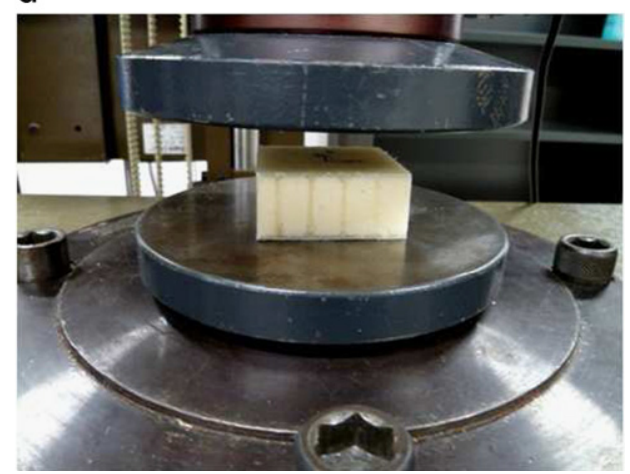

Fig. 1. Napco ${ }^{\mathbb{R}}$ sandwiches: (a) sandwich with foam core partly removed to show the transverse composite beams, (b) inclined through-thickness reinforcements, (c) needled facing fabric, (d) complete sample.

a

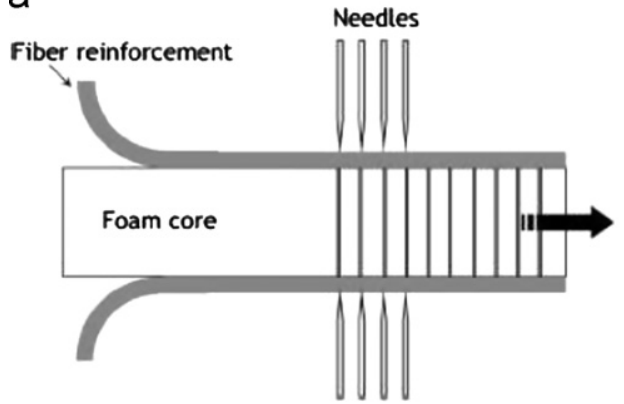

b

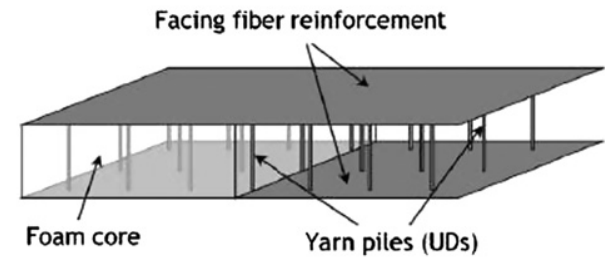

Fig. 2. Napco ${ }^{\circledR}$ technology [2]: (a) transverse needling, (b) 3D sandwich representation. 


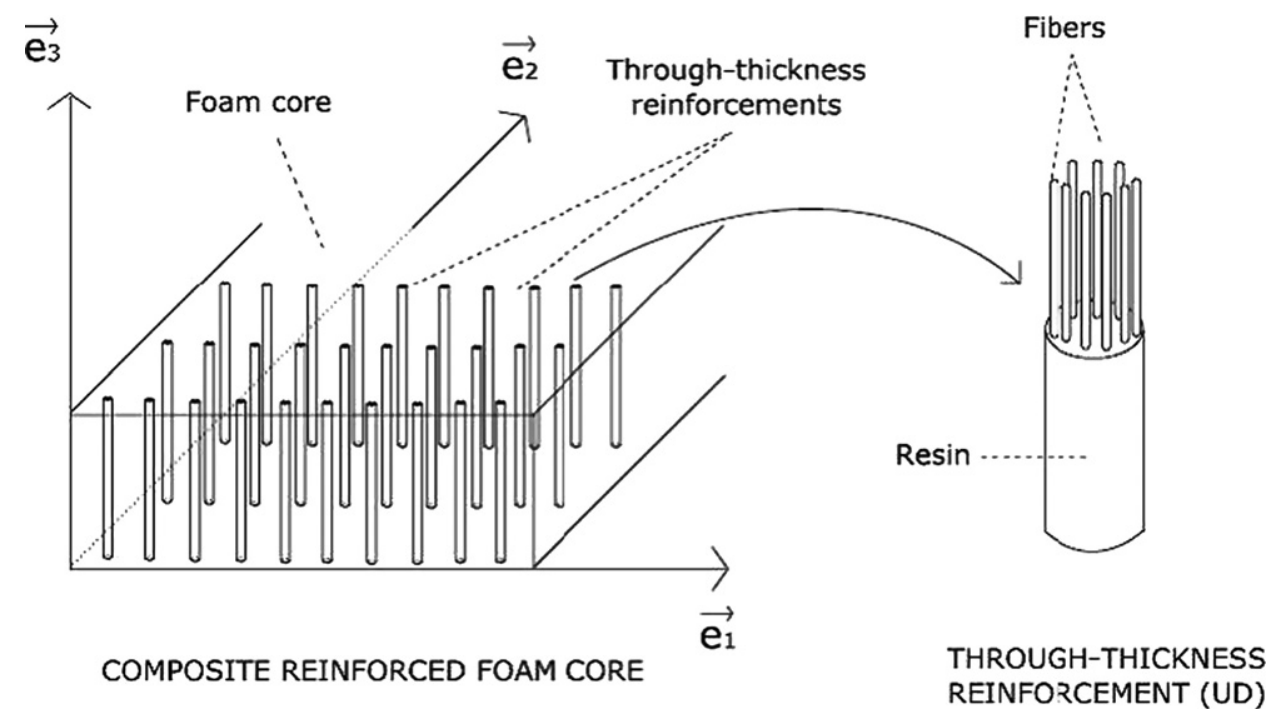

Fig. 3. Fiber reinforcements [2].

Table 2

Material parameters.

\begin{tabular}{lllll}
\hline Panel & $\mathrm{A}$ & $\mathrm{B}$ & $\mathrm{C}$ & $\mathrm{D}$ \\
\hline Reinforcements/dm & & & \\
$V_{f}(\%)$ & 69 & 138 & 276 & 415 \\
$E_{L}(\mathrm{MPa})$ & 4.01 & 2.63 & 3 & 1.93 \\
$v_{L T}$ & 6052.7 & 5098.8 & 5354.6 & 4615 \\
$G_{L T}(\mathrm{MPa})$ & 0.3448 & 0.3466 & 0.3461 & 0.3475 \\
$E_{T}(\mathrm{MPa})$ & 1308.4 & 1275.5 & 1284.3 & 1259.2 \\
$v_{T}$ & 3676.9 & 3558.5 & 3591.3 & 3493.4 \\
$E_{S}(\mathrm{MPa})$ & 0.4228 & 0.4065 & 0.4115 & 0.3957 \\
$v_{S}$ & 8463.3 & 8991.2 & 9181.7 & 8310.8 \\
\hline
\end{tabular}

Table 3

Geometric parameters.

\begin{tabular}{lllll}
\hline Panel & A & B & C & D \\
\hline Reinforcement radius (mm) & 1.1065 & 1.422 & 1.002 & 1.126 \\
Reinforcement volume fraction (\%) & 2.53 & 9 & 11.39 & 16.52 \\
Foam thickness (mm) & 20 & 20 & 20 & 20 \\
Skin thickness (mm) & 1.465 & 1.255 & 1.138 & 1.101 \\
\hline
\end{tabular}

$\bullet_{f}, \bullet_{r}, \bullet_{L}$ and $\bullet_{T}$ stand for the fibers, the resin, the longitudinal direction and the transverse direction. In order to simplify the geometric representation, the through-thickness reinforcing composites are supposed to have a constant circular section of radius $R$.

Four different types of needle pattern have been used to create different pile yarns (UDs) densities in the final sandwich structure. The material and geometric parameters of the four panels under consideration are summarized in Tables 2 and 3, respectively, where the subscript $\bullet_{s}$ stands for the skin parameters.

\subsection{Compression experiments}

Compression tests between parallel plates were performed on a material-testing machine (Zwick) mounted with a $100 \mathrm{kN}$-force cell. The panels have been characterized in through-thickness compression following the NF T 54-602 standard. The compression speed of the top platen was $2.2 \mathrm{~mm} \cdot \mathrm{min}^{-1}$. Ten samples with in-plane dimensions $50 \times 50 \mathrm{~mm}^{2}$ were tested for each of the four densities considered (see Fig. 1(d)). These dimensions are rather small but sufficiently large yet so that all the specimens contain many reinforcements, even in the case A where the volume fraction of reinforcements is very low. Although the specimens do not include a whole number of unit cells, which may act as representative volume elements, the volume fraction of reinforcements in the specimens coincides thus pretty much with the theoretical values of Table 3 .

Fig. 4 displays all the stress-displacement curves obtained from the experimental compression tests. The stress corresponds to the pressure applied on the top platen and the displacement is measured on the same platen in the loading direction.

For each density, the compression behavior of the composite material can be divided into three steps:

(i) First, as soon as the two parallel plates are in full contact with the lower and upper skins, the response curves are quasi-linear. It allows one to derive the overall longitudinal (through-thickness) Young's modulus of the corresponding sandwich structures. The little scatter of results is due to various geometric imperfections, especially regarding the shape of reinforcements and the variability of fiber volume fraction. It will further be shown that the buckling phenomenon that occurs during compression of the reinforced sandwich is mainly responsible for the imperfection sensitivity of the results.

(ii) The buckling phenomenon results in the loss of linearity of the stress-displacement curve and in a subsequent limit point. The corresponding limit stress is little scattered just like the constant slope during the initial elastic behavior, whereas the critical displacement (and therefore the critical deformation) does not seem to be sensitive to geometric imperfections. After reaching this limit point, the post-buckling behavior is clearly identified by a strong decrease of the stress level, presumably due to the successive failure of the reinforcements.

(iii) The stress finally tends towards a constant value, giving rise to a plateau. The corresponding stress level is very low and appears to be independent of the density of reinforcements. Indeed, at the final post-buckling stage, almost all the reinforcements are broken and the global response is governed by the foam core behavior, which is the same in all the panels considered.

\section{Analytical modeling of the compression behavior of Napco ${ }^{\circledR}$ sandwiches}

\subsection{Overall through-thickness longitudinal modulus}

Due to the elementary architecture of such reinforced sandwich structures with orthogonal reinforcements, simple 
a

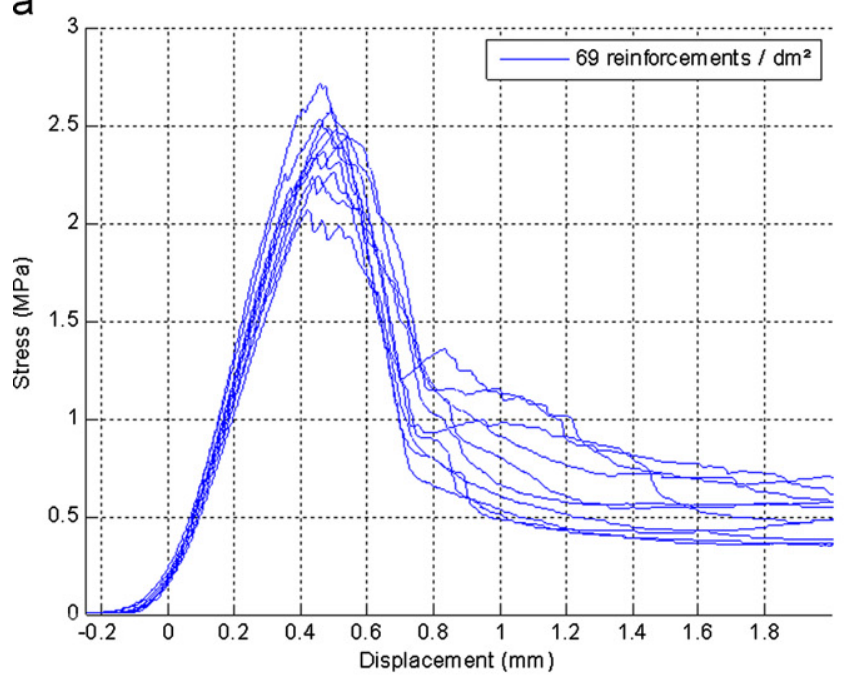

C

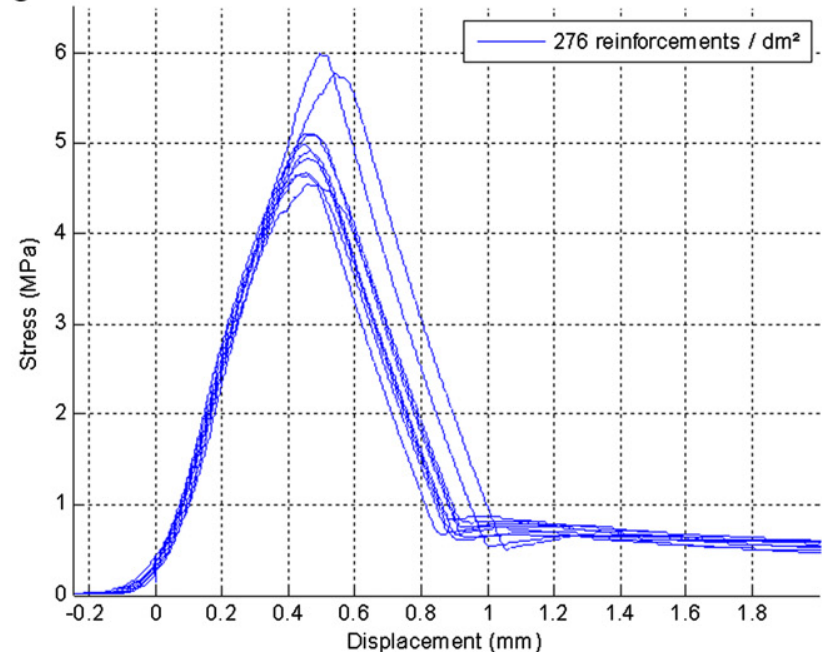

b

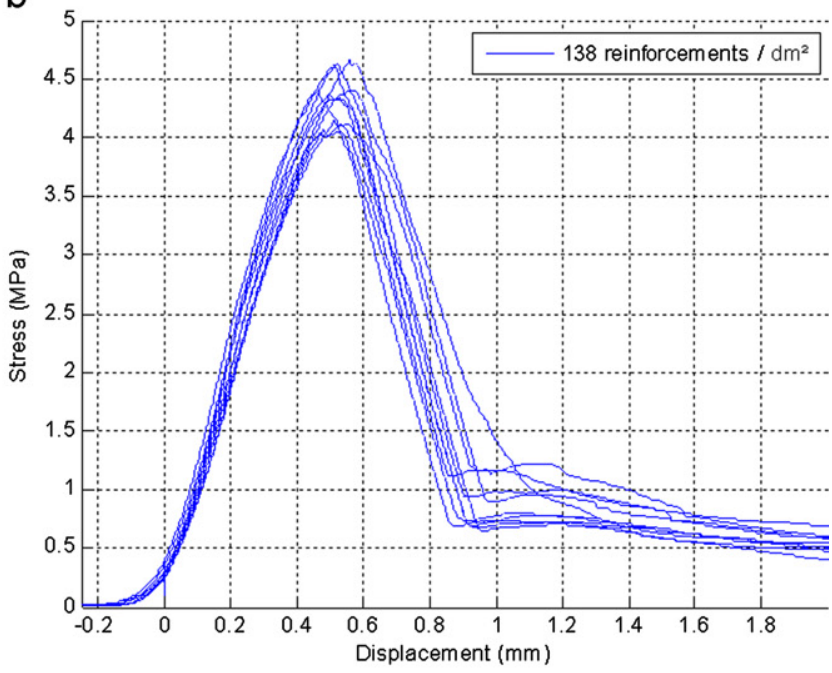

d

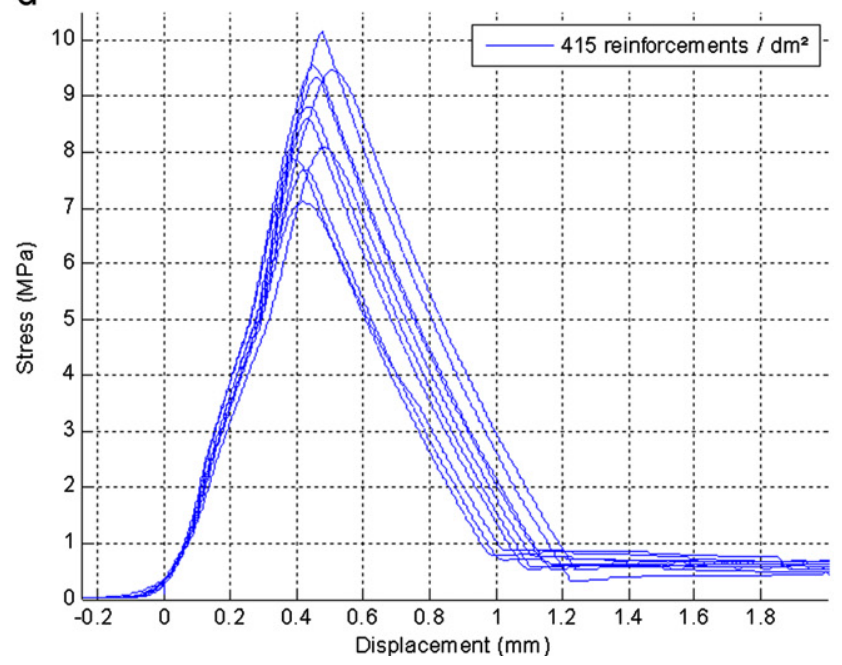

Fig. 4. Experimental stress-displacement curves from compression tests: (a) panel A, (b) panel B, (c) panel C, (d) panel D.

analytical expressions of the through-thickness Young's modulus can be easily derived by means of basic mixture laws. Considering first the reinforced foam core constituted by the core material and the cylindrical reinforcements in parallel, the Voigt upper bound is a good estimate of the equivalent modulus in the thickness direction:

$E_{r c}=E_{L} V_{f r}+E_{c}\left(1-V_{f r}\right)$

where $E_{c}$ is the foam core Young's modulus, $V_{f r}$ the volume fraction of fibrous reinforcements, and $E_{L}$ represents the longitudinal modulus of the reinforcements which has been preliminary calculated using Eq. (1). Next, the compression effective modulus of the whole sandwich can be assessed using the Reuss lower bound, due to the series configuration of the reinforced foam core and the skins:

$E_{e f f}=\frac{1}{\frac{V_{s}}{E_{s}}+\frac{1-V_{s}}{E_{\text {rc }}}}$

where $E_{s}$ and $V_{s}$ stand for Young's modulus and volume fraction of the skins, respectively.

Let us mention that, for validation purposes, this effective modulus $E_{\text {eff }}$ has also been calculated using finite element simulation. Contrary to the previous analytical approach, the numerical model takes into account the Poisson effect, but for
Table 4

Comparison between analytical and experimental effective moduli in the throughthickness direction.

\begin{tabular}{lcccc}
\hline Panel & A & B & C & D \\
\hline Analytical effective modulus (MPa) & 182.55 & 519.97 & \multicolumn{1}{c}{680.7} & 843.96 \\
Experimental mean value (MPa) & 132.28 & 226.93 & 287.81 & 459.93 \\
Experimental standard deviation (MPa) & 12 & 13.2 & 8.9 & 36.9 \\
Error (\%) & 27.5 & 56.4 & 57.7 & 45.5
\end{tabular}

all that, both methods lead to very similar numerical values (as it will be shown later). For the sake of clarity, only the analytical solutions are compared here with the experimental results for each sandwich panel (the experimental moduli are defined as the slope of the stress-strain curves in the first stage of deformation). Table 4 displays the analytical effective moduli and the experimental ones (mean values and standard deviations for the 10 specimens tested in each case) along with the relative error between both values.

As mentioned above, the analytical predictions for the effective modulus largely overestimate the experimental values. In light of all those observations, the determination of the buckling features of such reinforced sandwich structures under compression becomes relevant to improve their design. Through the 
evaluation of the critical loading, the bifurcation mode, and possibly the post-buckling behavior, it will be shown that the micro-buckling phenomenon is first responsible for the failure of the sandwich panels under such loading conditions.

\subsection{Analytical study of the buckling of a reinforced sandwich under through-thickness compression}

The main objective of this study is to find an analytical solution for the critical loading as well as the buckling mode of a reinforced sandwich under through-thickness compression, which will be compared to experimental results and observations, in order to validate the hypothesis according to which the collapse is mainly due to buckling and post-buckling. The critical loading complements all the effective moduli of the composite material that have already been analytically expressed, for dimensioning purposes.

\subsubsection{Problem definition}

Several assumptions must be made in order to be able to derive a closed-form expression for the critical value. First, a 2D simpler representation of the sandwich structure is preferred. The skins are then supposed to be stiff enough so that they do not deform during the compression experiment and do not influence the buckling behavior of the whole structure. Fig. 5 represents the model finally considered. The skins have been removed and will be replaced by proper boundary conditions. This unit cell (with two half-reinforcements and a foam block) is supposed to represent the global sandwich once the proper periodic boundary conditions are prescribed. This model is not capable of providing the so-called transverse buckling mode, as found by Parnes and Chiskis [6] in another context, due to the periodicity conditions. Only the shear buckling mode, which has shown to be predominant in previous studies [7], will be investigated in the sequel, according to the experimental observations.

The width $(2 t)$ of the composite reinforcements is chosen in such a way that their second moment-to-area ratio in the 2D model is equal to the one of the real cylindrical reinforcements in the 3D material $(t=R \sqrt{3} / 2)$. The same critical loading is thus obtained in both 2D and 3D configurations in the absence of foam, since the buckling load of a beam-like structure is governed by its bending rigidity. The width $(2 \mathrm{H})$ of the foam block is then defined in agreement with the volume fraction of reinforcements $\left(H=t\left(1-V_{f r}\right) / V_{f r}\right)$. Unlike the conclusions in Zhang and Latour [8], this particular choice will be proven to give satisfactory results. Finally, the global thickness $(2 L)$ is the real foam thickness measured experimentally.

The reinforcements (UDs) are assumed to behave like EulerBernoulli beams. Due to the entanglement of the fibers into the rigid skins, clamped boundary conditions are retained for the reinforcements instead of simply supported ones as supposed by Liu et al. [5]. Whereas the foam core is modeled by two horizontal

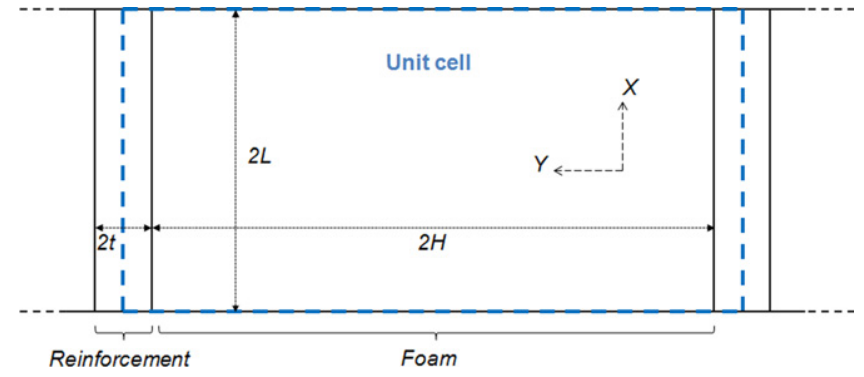

Fig. 5. 2D model for the analytical prediction of the buckling behavior. and vertical spring distributions in the latter reference, it is considered here as a 2D continuous solid.

The homogeneous and isotropic foam core is supposed to be linearly elastic (with Young's modulus $E_{c}$ and Poisson's ratio $v_{c}$ ). As for the transversely isotropic fibrous reinforcements, due to the kinematic hypotheses, only the longitudinal modulus $E_{L}$ will be involved in the sequel among all the elastic moduli defined in Eq. (1), so that the material can also be considered as isotropic.

Lastly, the unit cell is subjected to a compressive displacement in the thickness direction (like in experiment), which leads to buckling. The critical displacement and the associated bifurcation mode will be derived from a 3D framework: the theory is developed using a total Lagrangian formulation where the different components of the model are initially seen as 3D bodies [10-12].

\subsubsection{Theoretical formulation}

The critical loading $\lambda_{c r}$ and the bifurcation mode $\mathbf{X}$ of a 3D body are obtained by solving the following bifurcation equation:

$\forall \delta \mathbf{U}, \quad \int_{\Omega} \nabla^{T} \delta \mathbf{U}: \mathbf{K}\left(\lambda_{c r}\right): \nabla \mathbf{X} d \Omega=0$

where $\Omega$ is the volume of the 3D body in the reference configuration and $\delta \mathbf{U}$ stands for the variation of the displacement field in the body.

The fourth-order nominal tangent elastic tensor $\mathbf{K}$ can be written as follows:

$\mathbf{K}=\frac{\partial \boldsymbol{\Pi}}{\partial \mathbf{F}}=\mathbf{F} \cdot \frac{\partial \mathbf{\Sigma}}{\partial \mathbf{E}} \cdot \mathbf{F}^{T}+(\square \cdot \boldsymbol{\Sigma})^{T}=\mathbf{F} \cdot \mathbf{D} \cdot \mathbf{F}^{T}+(\llbracket \cdot \mathbf{\Sigma})^{T}$

In the above equation, $\mathbf{E}$ denotes the Green strain tensor and $\mathbf{\Sigma}$ the second Kirchhoff stress tensor (symmetric). $\mathbf{F}$ is the deformation gradient and $\boldsymbol{\Pi}=\mathbf{F} \cdot \boldsymbol{\Sigma}$ the first Kirchhoff stress tensor (nonsymmetric). $\llbracket$ represents the fourth-order unit tensor $\left(I_{i j k l}=\delta_{i l} \delta_{k j}\right)$ and the superscript ${ }^{T}$ the transposition of a second-order tensor and the major transposition of a fourth-order tensor $\left(\left(\mathbf{A}^{T}\right)_{i j k l}=A_{k l i j}\right)$, respectively. The fourth-order material tangent elastic tensor $\mathbf{D}$ of an isotropic material can be defined by its components in an orthonormal basis $D_{i j k l}=\Lambda \delta_{i j} \delta_{k l}+\mu\left(\delta_{i k} \delta_{j l}+\right.$ $\delta_{i l} \delta_{k j}$ ), where $\delta_{i j}$ is the Kronecker symbol, and $\Lambda$ and $\mu$ are the Lamé constants. Use is also made of Young's modulus $E$, Poisson's ratio $v$ and the shear modulus $G$ related to $\Lambda$ and $\mu$ by the standard relations $\Lambda=E v /(1+v)(1-2 v)$ and $\mu=G=E / 2(1+v)$.

We shall now derive more explicit expressions of the above tensors by exploiting the uniaxial stress state in the body. The uniform compressive displacement applied on the unit cell leads to compressive stresses in the reinforcements as well as in the foam core. Due to the respective moduli of the two materials, the uniaxial stresses in the pre-critical state are far larger in the reinforcements than in the foam, so that they will be neglected in the core material.

On one hand, the reinforcements are subjected to a nominal axial compressive stress $\Pi_{X X}=-P<0$ in their longitudinal direction, so that the first Kirchhoff stress tensor $\boldsymbol{\Pi}$ is expressed in the orthonormal basis $\left(\mathbf{e}_{X}, \mathbf{e}_{Y}, \mathbf{e}_{Z}\right)$ as

$\boldsymbol{\Pi}=-P \mathbf{e}_{X} \otimes \mathbf{e}_{X}=\left[\begin{array}{ccc}-P & 0 & 0 \\ 0 & 0 & 0 \\ 0 & 0 & 0\end{array}\right] \quad(P>0)$

Let us make the assumption that the pre-critical deformations are small, which is usually satisfied in practice:

$\|\nabla \mathbf{U}\| \ll 1$

Thus, the stress tensor $\boldsymbol{\Sigma}$ writes

$\boldsymbol{\Sigma}=\mathbf{F}^{-1} \cdot \mathbf{\Pi} \approx \boldsymbol{\Pi}$ 
The nominal tangent elastic tensor in Eq. (5) becomes then

$\mathbf{K} \approx \frac{\partial \mathbf{\Sigma}}{\partial \mathbf{E}}+(\square \cdot \mathbf{\Sigma})^{T}=\mathbf{D}-P \mathbf{e}_{i} \otimes \mathbf{e}_{X} \otimes \mathbf{e}_{X} \otimes \mathbf{e}_{i}$

which is independent of the spatial coordinates (the implicit summation convention on repeated indices is used with $i=X, Y, Z)$.

Furthermore, when dealing with 1D models like beams, ad hoc assumptions are usually added in order to enforce some specific stress state in the body. Namely, the transverse normal material stresses are assumed to be zero: $\Sigma_{Y Y}=\Sigma_{Z Z}=0$. Taking into account these assumptions leads one to replace tensor $\mathbf{D}$ with the reduced tensor $\mathbf{C}$ defined as

$C_{i j k l}=D_{i j k l}+\frac{D_{i j Y Y}\left(D_{Y Y Z Z} D_{Z Z k l}-D_{Z Z Z Z} D_{Y Y k l}\right)+D_{i j Z Z}\left(D_{Z Z Y Y} D_{Y Y k l}-D_{Y Y Y Y} D_{Z Z k l}\right)}{D_{Y Y Y Y} D_{Z Z Z Z}-D_{Y Y Z Z} D_{Z Z Y Y}}$

$(i, j) \neq(Y, Y),(Z, Z),(k, l) \neq(Y, Y),(Z, Z)$

It can be readily checked that tensor $\mathbf{C}$ has the major and both minor symmetries. In the sequel, we only need the following reduced moduli (and their equivalents obtained by major or minor symmetries):

$C_{X X X X}=E, \quad C_{X Y X Y}=C_{X Z X Z}=C_{Y Z Y Z}=G$

where only $E=E_{L}$ will explicitly appear in the final bifurcation equation.

On the other hand, as the existing initial stresses in the foam at the critical point have numerically shown to produce no significant effect on the buckling behavior, they are not introduced in our model, for simplicity purposes. Therefore, the nominal tangent elastic tensor in this case simply writes

$\mathbf{K} \approx \frac{\partial \mathbf{\Sigma}}{\partial \mathbf{E}}=\mathbf{D}$

The 2D model is supposed to reproduce the behavior of a panel with lateral dimensions much larger than thickness, so that the plane strain hypothesis is adopted. Thus, tensor $\mathbf{D}$ has no need to be reduced and only the plane components will be used in the sequel:

$D_{X X X X}=D_{Y Y Y Y}=\Lambda_{c}+2 \mu_{c}, \quad D_{X X Y Y}=\Lambda_{c}, \quad D_{X Y X Y}=\mu_{c}$

and their equivalents obtained by major or minor symmetries.

Eventually, the bifurcation equation (4) of the unit cell writes

$$
\begin{aligned}
& \forall \delta \mathbf{U}_{f r}, \delta \mathbf{U}_{c}, \quad \int_{\Omega_{f r}} \nabla^{T} \delta \mathbf{U}_{f r}:\left(\mathbf{C}-P_{c r} \mathbf{e}_{i} \otimes \mathbf{e}_{X} \otimes \mathbf{e}_{X} \otimes \mathbf{e}_{i}\right): \nabla \mathbf{X}_{f r} d \Omega_{f r} \\
& \quad+\int_{\Omega_{c}} \nabla^{T} \delta \mathbf{U}_{c}: \mathbf{D}: \nabla \mathbf{X}_{c} d \Omega_{c}=0
\end{aligned}
$$

The compressive stress $(P>0)$ is related to the enforced displacement $\lambda>0$ (which will act as the bifurcation parameter) by the following relation:

$P=\frac{E_{L} \lambda}{2 L}$

and $\mathbf{U}_{f r}, \mathbf{U}_{c}, \mathbf{X}_{f r}$ and $\mathbf{X}_{c}$ represent the displacement field and bifurcation mode components, respectively, relative to the fibrous reinforcements (with indice $\bullet_{\text {fr }}$ ) and the foam core (with indice $\bullet_{c}$ ).

Let us now consider the bending problem of a reinforcement in the $X Y$-plane. The Euler-Bernoulli beam theory is employed, as transverse shear effects may be negligible in practice. The EulerBernoulli kinematics is defined by two scalar displacement fields $U(X)$ and $V(X)$, respectively the axial and transverse displacements of the centroid axis of the beam. When the beam buckles from the straight position (the fundamental solution) to a bent shape, the expressions for the bifurcation mode $\mathbf{X}_{f r}$ and the displacement variation $\delta \mathbf{U}_{f r}$ are both chosen according to the
Euler-Bernoulli kinematics:

$\mathbf{X}_{f r}=\left|\begin{array}{ll}\mathcal{U}_{f r}-Y \mathcal{V}_{f r}, X, \\ \mathcal{V}_{f r},\end{array} \quad \delta \mathbf{U}_{f r}=\right| \begin{aligned} & \delta U_{f r}-Y \delta V_{f r}, X \\ & \delta V_{f r} \\ & 0\end{aligned}$

The same fields can be used for both half-reinforcements in the unit cell, due to the enforced periodicity conditions, so that the two half-beams can be identified as a single entire beam.

On the other side, the bifurcation mode $\mathbf{X}_{c}$ and the displacement variation $\delta \mathbf{U}_{c}$ in the foam core are classically expressed in the orthonormal basis $\left(\mathbf{e}_{X}, \mathbf{e}_{Y}, \mathbf{e}_{Z}\right)$ :

$\mathbf{X}_{c}=\mid \begin{array}{ll}\mathcal{U}_{c}, \\ \mathcal{V}_{c}, & \delta \mathbf{U}_{c}=\mid \begin{array}{l}\delta U_{c} \\ 0,\end{array} \\ \delta V_{c} \\ 0\end{array}$

The global bifurcation equation then writes

$$
\begin{aligned}
\forall & \delta U_{f r}, \delta V_{f r}, \delta U_{c}, \delta V_{c}, \quad \int_{\Omega_{f r}}\left[E_{L}\left(\mathcal{U}_{f r}, X-Y \mathcal{V}_{f r}, X X\right)\left(\delta U_{f r}, X-Y \delta V_{f r}, X X\right)\right. \\
& \left.-\frac{E_{L} \lambda_{c r}}{2 L}\left(\mathcal{U}_{f r}, X-Y \mathcal{V}_{f r}, X X\right)\left(\delta U_{f r}, X-Y \delta V_{f r}, X X\right)-\frac{E_{L} \lambda_{c r}}{2 L} \mathcal{V}_{f r}, X \delta V_{f r, X}\right] d \Omega_{f r} \\
& +\int_{\Omega_{c}}\left(D_{X X X X} \mathcal{U}_{c, X} \delta U_{c, X}+D_{X X Y Y} \mathcal{V}_{c, Y} \delta U_{c, X}+D_{Y Y X X} \mathcal{U}_{c, X} \delta V_{c, Y}+D_{Y Y Y Y} \mathcal{V}_{c, Y} \delta V_{c, Y}\right. \\
& \left.+D_{X Y X Y} \mathcal{V}_{c, X} \delta U_{c, Y}+D_{X Y Y X} \mathcal{U}_{c, Y} \delta U_{c, Y}+D_{Y X X Y} \mathcal{V}_{c, X} \delta V_{c, X}+D_{Y X Y X} \mathcal{U}_{c, Y} \delta V_{c, X}\right) d \Omega_{c}=0
\end{aligned}
$$

where $Y$ stands for the $Y$-coordinate of a current point relative to the centroid axis of the corresponding zone.

First, integrating over the cross-section of the beam, then integrating by parts with respect to $X$ and $Y$, and eliminating negligible higher-order terms (presupposing that $\lambda_{c r} \ll L$ ) yields four local partial differential equations for the components $\mathcal{U}_{f r}$, $\mathcal{V}_{f r}, \mathcal{U}_{c}$ and $\mathcal{V}_{c}$ of the eigenmode:

$$
\begin{aligned}
& 2 E_{L} t \mathcal{U}_{f r, X X}+\left.\mu_{c}\left(\mathcal{U}_{c, Y}+\mathcal{V}_{c, X}\right)\right|_{Y=-H}-\left.\mu_{c}\left(\mathcal{U}_{c}, Y+\mathcal{V}_{c, X}\right)\right|_{Y=H}=0 \\
& \frac{2 E_{L} t^{3}}{3} \mathcal{V}_{f r}, X X X X+\frac{E_{L} t \lambda_{c r}}{L} \mathcal{V}_{f r}, X X+\Lambda_{c} \mathcal{U}_{c},\left.X\right|_{Y=H}-\Lambda_{c} \mathcal{U}_{c},\left.X\right|_{Y=-H} \\
& \quad+\left.\left(\Lambda_{c}+2 \mu_{c}\right) \mathcal{V}_{c, Y}\right|_{Y=H}-\left.\left(\Lambda_{c}+2 \mu_{c}\right) \mathcal{V}_{c, Y}\right|_{Y=-H} \\
& \quad-\left.t \mu_{c}\left(\mathcal{U}_{c}, X Y+\mathcal{V}_{c}, X X\right)\right|_{Y=-H}-\left.t \mu_{c}\left(\mathcal{U}_{c}, X Y+\mathcal{V}_{c}, X X\right)\right|_{Y=H}=0 \\
& \left(\Lambda_{c}+2 \mu_{c}\right) \mathcal{U}_{c, X X}+\mu_{c} \mathcal{U}_{c}, Y Y+\left(\Lambda_{c}+\mu_{c}\right) \mathcal{V}_{c, X Y}=0 \\
& \left(\Lambda_{c}+2 \mu_{c}\right) \mathcal{V}_{c, Y Y}+\mu_{c} \mathcal{V}_{c, X X}+\left(\Lambda_{c}+\mu_{c}\right) \mathcal{U}_{c, X Y}=0
\end{aligned}
$$

The last two Eqs. (19c) and (19d) identify with classical local equilibrium equations of the foam block in a 2D framework. Similarly, the first two Eqs. (19a) and (19b) look like the classical buckling differential equations of a beam, but include new quantities characterizing the influence of stresses at the interface between reinforcements and foam during the buckling phenomenon. Let us mention that these additional terms are here naturally obtained through integrations by parts performed in the foam block.

At this stage, one has to specify the boundary conditions in order to solve the previous system. First, connecting conditions for the displacement fields (bifurcation mode) must be satisfied at the interfaces between the foam core and reinforcements, namely:

$$
\begin{aligned}
& \mathcal{U}_{f r}-t \mathcal{V}_{f r}, X-\left.\mathcal{U}_{c}\right|_{Y=-H}=0 \\
& \mathcal{U}_{f r}+t \mathcal{V}_{f r}, X-\left.\mathcal{U}_{c}\right|_{Y=H}=0 \\
& \mathcal{V}_{f r}-\left.\mathcal{V}_{c}\right|_{Y=-H}=0 \\
& \mathcal{V}_{f r}-\left.\mathcal{V}_{c}\right|_{Y=H}=0
\end{aligned}
$$

The two ends of the reinforcements are clamped in the rigid skins. Due to the possible relative translation of the skins, only guided boundary conditions are retained for both ends and lead to the following kinematical constraints: $\mathcal{V}_{f r, X}(-L)=\mathcal{V}_{f r}, X(L)=0$. Taking into account $\delta V_{f r}, X(-L)=\delta V_{f r}, X(L)=0$ in the bifurcation equation (18) 
leads one, after integration by parts, to the remaining stress boundary conditions at the ends $X=-L$ and $X=L: 2 E_{L} t \mathcal{U}_{f r}, X( \pm L)=0$ and $\left(2 E_{L} t^{3} / 3\right) \mathcal{V}_{f r}, X X X( \pm L)+\left(E_{L} t \lambda_{c r} / L\right) \mathcal{V}_{f r}, X( \pm L)-t \mu_{c}\left(\mathcal{U}_{c, Y}( \pm L,-H)+\right.$ $\left.\mathcal{V}_{c, X}( \pm L,-H)\right)-t \mu_{c}\left(\mathcal{U}_{c}, Y( \pm L, H)+\mathcal{V}_{c}, X( \pm L, H)\right)=0$. Some additional kinematical constraints must be enforced in order to prevent the unit cell from possible rigid modes. For this purpose, the displacement of the mid-point $(X=0)$ of the reinforcements is assumed to be null: $\mathcal{U}_{f r}(0)=\mathcal{V}_{f r}(0)=0$.

The last boundary conditions refer to the interfaces between the foam core and the skins which are not represented in the present model. The skins are supposed to be sufficiently rigid so that both modal displacements can be considered as constant along the interfaces, and the corresponding displacement boundary conditions thus write

$$
\begin{aligned}
& \left.\mathcal{U}_{C}\right|_{X=-L}=\left.\mathcal{U}_{f r}\right|_{X=-L} \\
& \left.\mathcal{U}_{C}\right|_{X=L}=\left.\mathcal{U}_{f r}\right|_{X=L} \\
& \left.\mathcal{V}_{c}\right|_{X=-L}=\left.\mathcal{V}_{f r}\right|_{X=-L} \\
& \left.\mathcal{V}_{C}\right|_{X=L}=\left.\mathcal{V}_{f r}\right|_{X=L}
\end{aligned}
$$

To make possible the analytical resolution of the problem, the last two equations will be sooner replaced by the corresponding stress boundary conditions (as if these two edges were free in the Y-direction):

$$
\begin{aligned}
& \left.\mu_{c}\left(\mathcal{U}_{c}, Y+\mathcal{V}_{c}, X\right)\right|_{X=-L}=0 \\
& \left.\mu_{c}\left(\mathcal{U}_{c}, Y+\mathcal{V}_{c, X}\right)\right|_{X=L}=0
\end{aligned}
$$

This alternative will allow us to derive a closed-form solution with a very good accuracy, since reinforcements are not too distant from each other.

\subsubsection{Solution procedure}

The bifurcation mode of a single Euler-Bernoulli beam under axial compression with the boundary conditions defined above takes the following form:

$$
\left\{\begin{array}{l}
\mathcal{U}=0 \\
\mathcal{V}=\sin \frac{\pi X}{2 L}
\end{array}\right.
$$

Considering that the foam all around the reinforcements does not affect the buckling mode components of the beam, the following assumptions are made:

$$
\left\{\begin{array}{l}
\mathcal{U}_{f r}=0 \\
\mathcal{V}_{f r} \propto \sin \frac{\pi X}{2 L}
\end{array}\right.
$$

which are consistent with the corresponding boundary conditions.

Concerning the foam displacement field, a separation of variables is performed and the following forms are presupposed, according to Eq. (24):

$\left\{\begin{array}{l}\mathcal{U}_{c}=\alpha(Y) \cos \frac{\pi X}{2 L} \\ \mathcal{V}_{c}=\beta(Y) \sin \frac{\pi X}{2 L}\end{array}\right.$

where $\alpha(Y)$ and $\beta(Y)$ are unknown functions to be determined.

Solving the two equilibrium equations in the foam block (19c) and (19d), together with the four connecting conditions (20), leads to the following bifurcation mode:

$$
\left\{\begin{array}{l}
\mathcal{U}_{f r}=0 \\
\mathcal{V}_{f r}=\left(K_{1} \cosh \frac{\pi H}{2 L}+K_{2} H \sinh \frac{\pi H}{2 L}\right) \sin \frac{\pi X}{2 L} \\
\mathcal{U}_{c}=\left(K_{3} \sinh \frac{\pi Y}{2 L}+K_{2} Y \cosh \frac{\pi Y}{2 L}\right) \cos \frac{\pi X}{2 L} \\
\mathcal{V}_{c}=\left(K_{1} \cosh \frac{\pi Y}{2 L}+K_{2} Y \sinh \frac{\pi Y}{2 L}\right) \sin \frac{\pi X}{2 L}
\end{array}\right.
$$

with

$K_{1}=2 L \pi H \cosh \frac{\pi H}{2 L}+\left(4 L^{2}\left(3-4 v_{c}\right)-t H \pi^{2}\right) \sinh \frac{\pi H}{2 L}$

$K_{2}=t \pi^{2} \cosh \frac{\pi H}{2 L}-2 L \pi \sinh \frac{\pi H}{2 L}$

$K_{3}=2 L \pi\left(H+3 t-4 t v_{c}\right) \cosh \frac{\pi H}{2 L}-t H \pi^{2} \sinh \frac{\pi H}{2 L}$

The remaining boundary conditions and the first local equation (19a) are automatically verified. Finally, Eq. (19b) can be solved and leads to the critical displacement:

$$
\begin{aligned}
\lambda_{c r}= & \left(\left[24 L^{2} E_{c}\left(4 L^{2}+t^{2} \pi^{2}\right)\left(v_{c}-1\right)\right] \cosh ^{2} \frac{\pi H}{2 L}+\left[2 E_{L} t^{3} \pi^{3} L\left(4 v_{c}^{2}+v_{c}-3\right)\right.\right. \\
& \left.+48 L^{3} E_{c} t \pi\left(2 v_{c}-1\right)\right] \cosh \frac{\pi H}{2 L} \sinh \frac{\pi H}{2 L}+96 L^{4} E_{c}\left(1-v_{c}\right)-E_{L} t^{3} \pi^{4} H\left(1+v_{c}\right) \\
& \left.-24 L^{2} E_{c} t H \pi^{2}\right) /\left(6 E_{L} t L \pi\left(1+v_{c}\right)\left[2 L\left(4 v_{c}-3\right) \cosh \frac{\pi H}{2 L} \sinh \frac{\pi H}{2 L}-\pi H\right]\right)
\end{aligned}
$$

\section{Numerical and experimental validation}

4.1. Numerical computation of the critical displacement, bifurcation mode and post-buckling behavior

Three-dimensional numerical finite element computations have been performed in order to validate the previous analytical solution. First, a linearized buckling analysis is carried out. An hexagonal arrangement of the reinforcements has been retained, according to the experimental patterns. Thus, the overall mechanical response of the composite reinforced foam core (and consequently of the sandwich) is transversely isotropic. A 3D unit cell is only considered, for efficiency purposes, but here including the skins and the full material properties of the transversely isotropic reinforcements. The geometry of the plane-parallel unit cell and the associated finite element mesh (made up of 20-noded hexahedral elements) are depicted in Fig. 6. Periodic boundary conditions are enforced on the lateral faces of the unit cell in both directions, what makes the buckling behavior similar to that of an infinite sandwich plate. Lastly, the bottom and top faces of the sandwich cell are, respectively, subjected to zero and non-zero (negative) displacement boundary conditions in the throughthickness direction, in order to compress the composite and give rise to the expected buckling phenomenon.

In order to better compare experimental and modeling results, incremental post-buckling calculations are carried out. It will allow us to recover first the initial pre-buckling stiffnesses in the through-thickness direction, re-estimate the critical buckling displacements and further analyze the post-buckling behavior. A little defect is added to the model (a small concentrated load along one axis of the sandwich plane) in order to trigger the buckling and post-buckling phenomena.

Secondarily, 2D finite element linearized buckling analyses have also been performed (including the skins as well) in order to evaluate the relative influence of different hypotheses (2D representation, boundary conditions) on the critical value. 
a

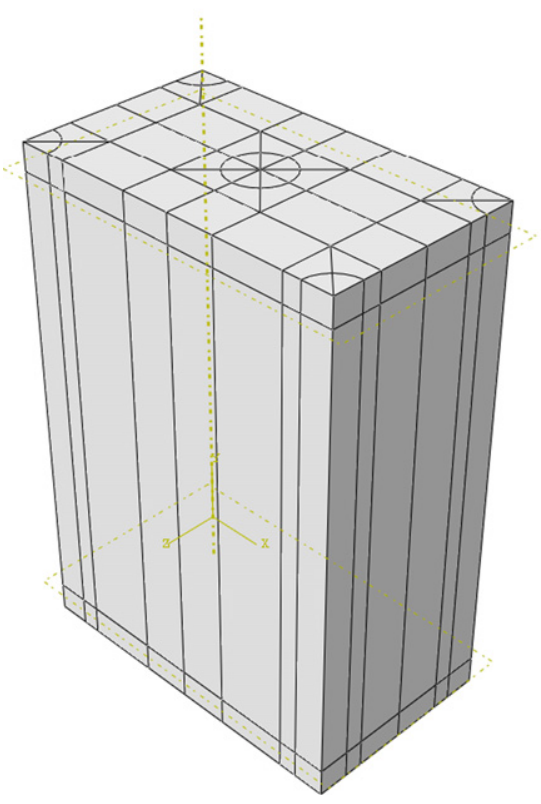

b

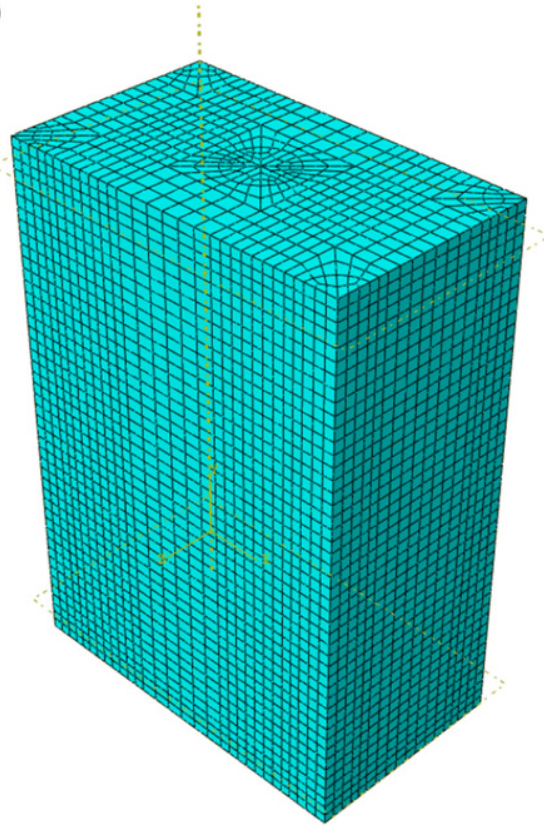

Fig. 6. Model for the 3D finite element computations: (a) 3D unit cell, (b) global mesh.

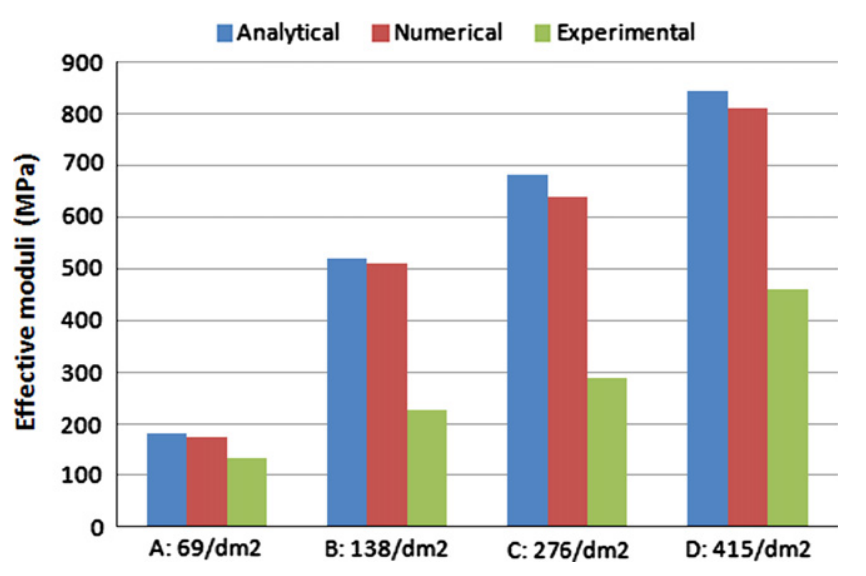

Fig. 7. Comparison between analytical, numerical and experimental effective moduli in the through-thickness direction.

\subsection{Comparison between analytical solutions and numerical/ experimental results}

First, for each panel, the initial slope of the numerical stressstrain curves obtained during the incremental simulations are compared to both analytical effective moduli and experimental corresponding mean values (see Fig. 7). As expected, the numerical moduli only differ a little from the analytical ones, due to the Poisson effect. It corroborates the idea that experimental moduli are far less than predicted theoretical values.

For each density, the critical displacement has been then determined in an analytical way (Eq. (28)), and by means of numerical calculations (2D and 3D linearized buckling analyses). All these results are summarized in Table 5 and displayed in Fig. 8. The critical values predicted by the $2 \mathrm{D}$ and 3D numerical analyses are very similar for all the density range (with a relative error less than 6\%). This result validates the hypotheses retained when defining a 2D model equivalent to the real 3D geometry. Moreover, the present analytical solution is shown to be in good accordance with numerical results, at least for the three higher
Table 5

Comparison between analytical, 2D and 3D numerical critical displacements.

\begin{tabular}{lllll}
\hline Panel & A & B & C & D \\
\hline Analytical solution (mm) & 0.2658 & 0.354 & 0.2298 & 0.2532 \\
2D FE model (mm) & 0.53578 & 0.38989 & 0.24204 & 0.25659 \\
3D FE model (mm) & 0.55887 & 0.40541 & 0.25773 & 0.27366 \\
\hline
\end{tabular}

densities of reinforcements (panels B, C and D). In these three cases, the agreement is better (less than $12 \%$ of error) than it is with most of other estimations of the critical value in the literature. For example, Rosen's formula, initially dedicated to the micro-buckling of fiber-reinforced composite materials, is not suitable in this context, except perhaps for the most dilute case (panel A) where it gives a better result than ours. Modified expressions of Rosen's formula, such as the one derived by Drapier et al. [4], are most likely to agree with numerical results, even if the related hypotheses (simplified expressions of the deformation field in the foam core) are not properly justified and cannot comply with most of the boundary and continuity conditions. Lastly, the general solution obtained by Liu et al. [5] for sandwich beams with pin-reinforced foam cores is difficult to compare with our own solution, since there are many differences between the two approaches (in [5], the pins are simply supported instead of clamped, it is not clear whether the unit cell is designed in $2 \mathrm{D}$ or $3 \mathrm{D}$, and the definition of the foundation modulus is not straightforward). The main difference between our analytical solution and the 2D finite element model lies in the consideration of the skins in the latter. Approximated boundary conditions have been retained in the analytical approach, that do not match the real conditions in the presence of skins, in order to simplify the analytical resolution and make possible the achievement of a closed-form expression for the critical displacement. Considering the numerical values in Table 5 , this choice of boundary conditions is not so detrimental, as soon as the volume fraction of reinforcements is about $10 \%$ or higher. On the contrary, with the smallest density (panel A), a large discrepancy is noticed between the analytical and numerical predictions, what points out the limitations of the present analytical model. When fibrous 
reinforcements are much less numerous and very distant from each other, the skin effect becomes more and more apparent in the bifurcation mode shape and thus in the critical displacement value.

Further, the post-buckling behavior is investigated through the comparison between the numerical stress-displacement curves and the experimental ones (Fig. 9). In the first stage of deformation, the difference between theoretical and experimental initial stiffnesses is clearly emphasized. It is due to unavoidable imperfections related to the Napco ${ }^{\mathbb{R}}$ manufacturing process. Then, the analytical critical displacements are compared to the stressdisplacement curves. The analytical solution seems to coincide fairly well with the points in both numerical and experimental

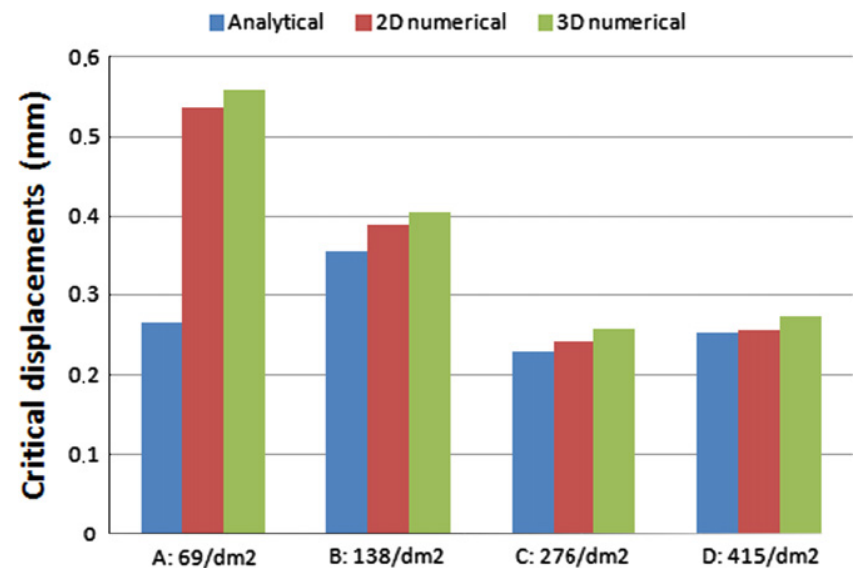

Fig. 8. Critical displacements obtained from analytical and numerical (2D/3D) analyses.

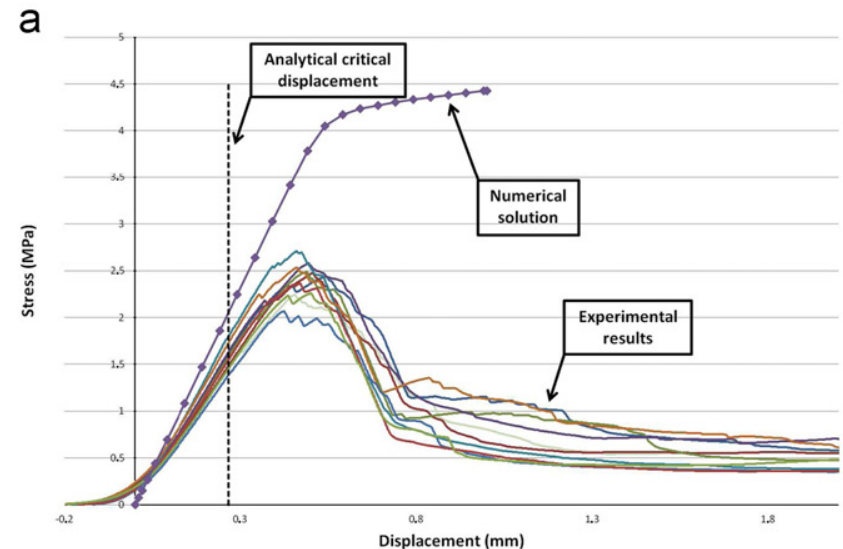

C

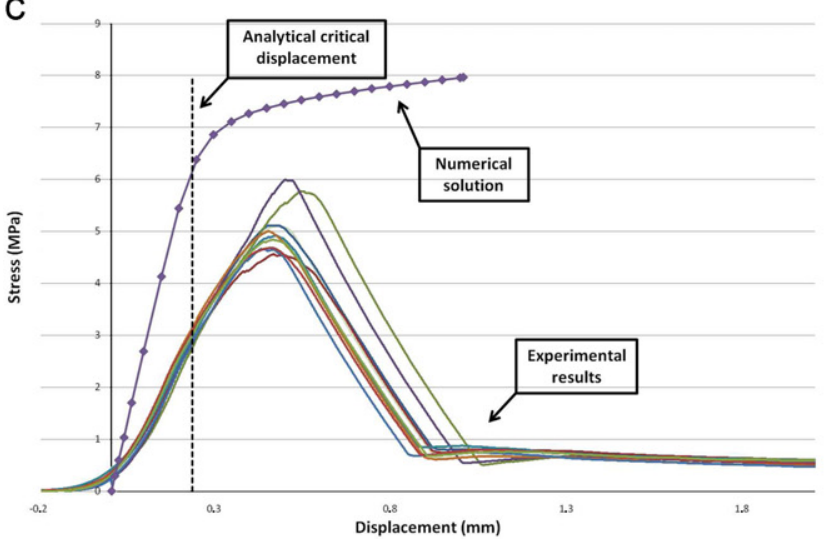

curves corresponding to the loss of linearity, except for the very small density (panel A) for the reasons discussed above. For each panel, these points have almost always the same abscissa (displacement), whatever the experimental curve considered. In the post-buckling range, the numerical curve always overestimates the experimental ones. No limit point is observed numerically (as if to say the post-buckling behavior is stable) whereas all the experimental curves display a snap-through phenomenon. These limit points and the subsequent great decrease of the stress level are certainly due to secondary effects, such as the successive failure of fibers and reinforcements during the post-buckling stage.

Finally, Figs. 10 and 11 display the bifurcation modes obtained by both 2D and 3D linearized buckling analyses and the postbuckled shape observed experimentally. The identical deformation shapes, obtained from both numerical and experimental points of view, confirm the predominance of the micro-buckling phenomenon in the mechanical behavior of such sandwiches under through-thickness compression.

\section{Conclusions}

The Napco ${ }^{\mathbb{R}}$ technology is a patented process that transversally strengthens the foam core of a sandwich structure with fiber yarns taken from facings. In this study, we investigated the potential of such a reinforced sandwich in its through-thickness compression behavior. The main issue is the large discrepancy observed between the analytical predictions of the effective moduli using classical mixture laws and the experimental responses, due to many imperfections. The objective of the present work was to demonstrate that micro-buckling is mainly

b

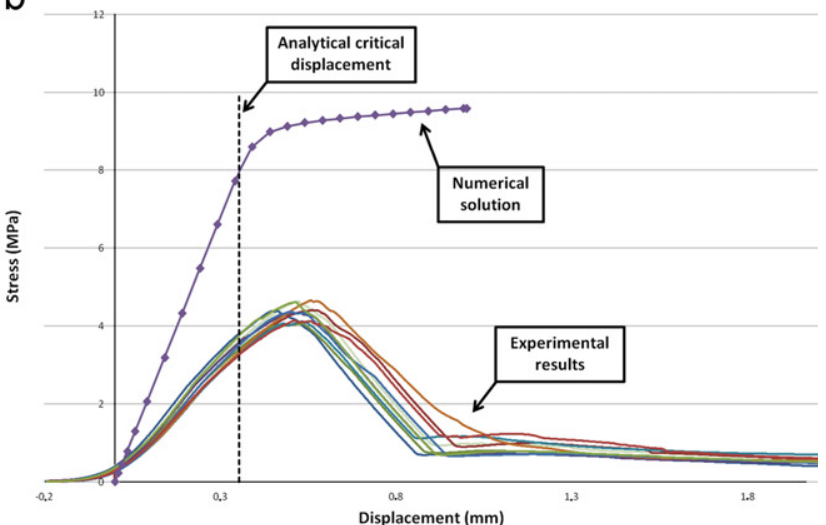

d

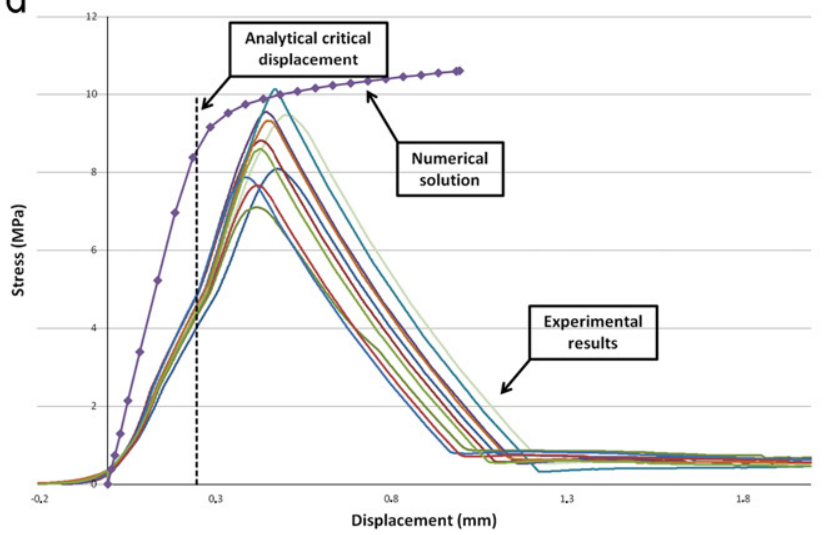

Fig. 9. Comparison between numerical and experimental stress-displacement curves: (a) panel A, (b) panel B, (c) panel C, (d) panel D. 

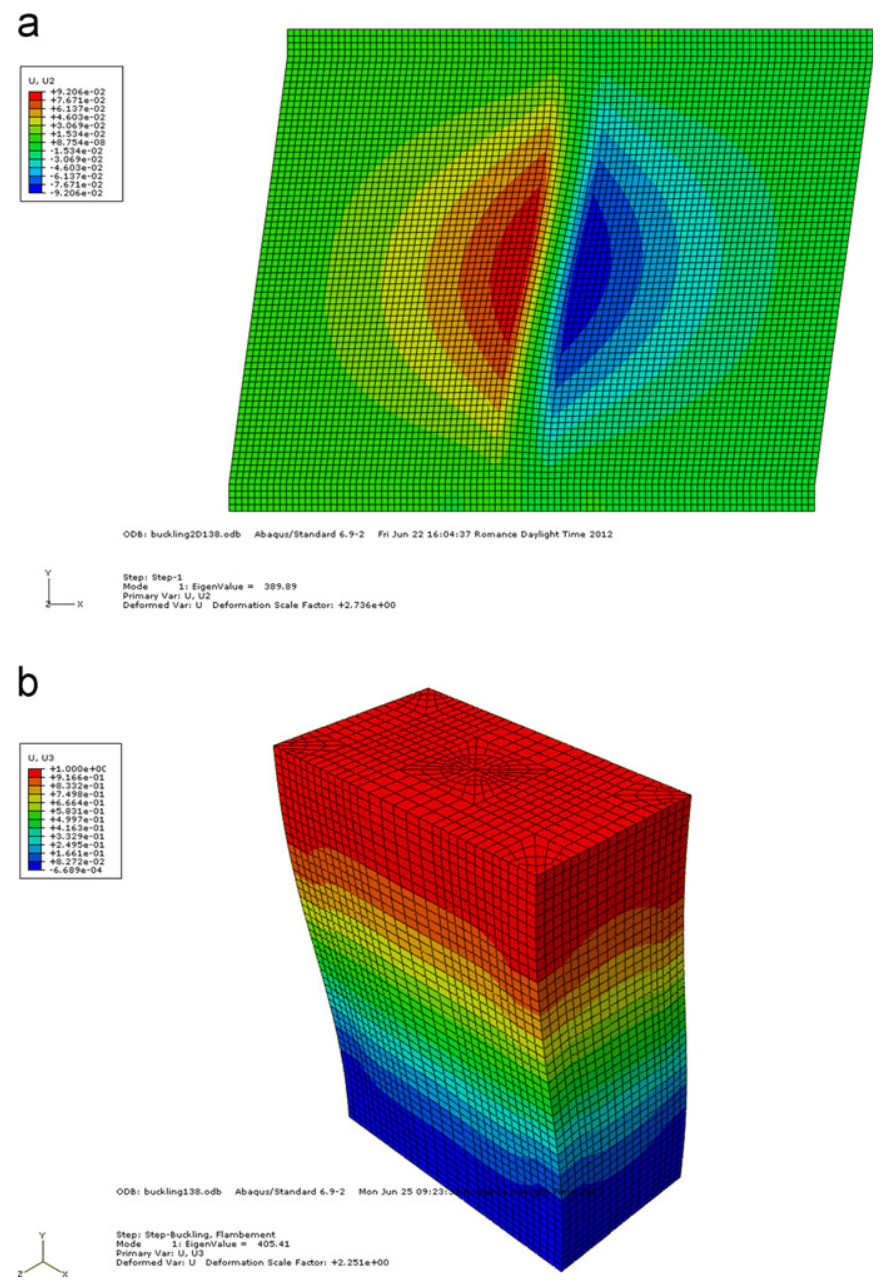

Fig. 10. Numerical bifurcation modes: (a) 2D model, (b) 3D model.

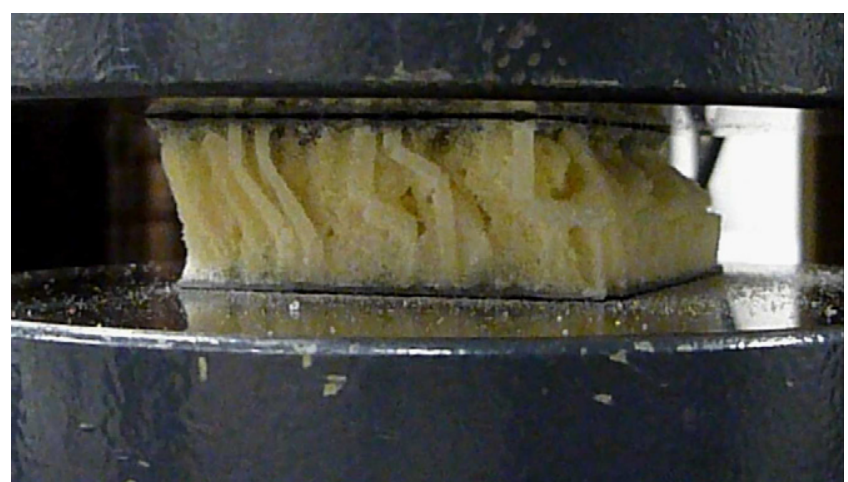

Fig. 11. Post-buckled deformation shape during experimental compression test.

responsible for the collapse of such sandwich structures under through-thickness compression. An analytical closed-form solution for the critical displacement has been proposed, deriving from a 3D bifurcation analysis. A 2D model was defined, for simplicity purposes, only considering a unit cell of the reinforced foam core (due to the material periodicity), with appropriate boundary conditions to consider the influence of the skins. The reinforcements were assumed to behave like Euler-Bernoulli beams whereas the foam core was modeled as a 2D continuous solid, without considering any simplified deformation field. The 2D and 3D finite element models of a unit cell have also been developed, for validation purposes. It will further allow to generalize the buckling analysis to other configurations (for instance, other distributions and/or orientations of the throughthickness reinforcements) where an analytical solution is no more available. The 2D and 3D models are related in such a way that the second moment-to-area ratio of the beams and the volume fraction of the reinforcements remain unchanged.

Experimental compression tests have been performed for four different sandwich panels (with various densities of reinforcements). Comparisons between analytical/numerical predictions and experiments were discussed and clearly showed the accuracy of the present analytical model. The closed-form expression of the critical displacement is shown to be suitable to properly predict the micro-buckling behavior of such sandwiches, as long as the volume fraction of reinforcements is sufficiently high, say greater than $10 \%$. The local buckling of the reinforced sandwiches is thus proved to be the initial reason for the collapse in throughthickness compression.

\section{Acknowledgments}

The authors are indebted to the French Ministry of Economy, Finance and Industry (NWC-X Project, Contract no. 09290 6242) for its financial support.

\section{References}

[1] Lascoup B, Aboura Z, Khellil K, Benzeggagh ML. On the mechanical effect of stitch addition in sandwich panel. Compos Sci Technol 2006;66(10):1385-98.

[2] Guilleminot J, Comas Cardona S, Kondo D, Binetruy C, Krawczak P. Multiscale modelling of the composite reinforced foam core of a 3D sandwich structure. Compos Sci Technol 2008;68(7-8):1777-86.

[3] Lascoup B, Aboura Z, Khellil K, Benzeggagh ML. Prediction of out-of-plane behavior of stitched sandwich structure. Compos Part B: Eng 2012;43(8):2915-20.

[4] Drapier S, Gardin C, Grandidier JC, Potier-Ferry M. Structure effect and microbuckling. Compos Sci Technol 1996;56(7):861-7.

[5] Liu T, Deng ZC, Lu TJ. Analytical modeling and finite element simulation of the plastic collapse of sandwich beams with pin-reinforced foam cores. Int J Solids Struct 2008;45(18-19):5127-51.

[6] Parnes R, Chiskis A. Buckling of nano-fibre reinforced composites: a reexamination of elastic buckling. J Mech Phys Solids 2002;50(4):855-79.

[7] Zhang G, Latour RA. An analytical and numerical study of fiber microbuckling. Compos Sci Technol 1994;51(1):95-109.

[8] Zhang G, Latour RA. A three-dimensional micromechanical model of the compressive behavior of unidirectional FRP composites. J Thermoplastic Compos Mater 1997;10(2):173-84.

[9] Berthelot JM. Matériaux Composites: Comportement Mécanique et Analyse des Structures. Masson; 1996.

[10] Le Grognec P, Le van A. Some new analytical results for plastic buckling and initial post-buckling of plates and cylinders under uniform compression. Thin-Walled Struct 2009;47(8-9):879-89.

[11] Le Grognec P, Le van A. On the plastic bifurcation and post-bifurcation of axially compressed beams. Int J Non-Linear Mech 2011;46(5):693-702.

[12] Le Grognec P, Nguyen QH, Hjiaj M. Exact buckling solution for two-layer Timoshenko beams with interlayer slip. Int $\mathrm{J}$ Solids Struct 2012;49(1):143-50. 